\section{MHC-I SKEWING IN MUTANT CALRETICULIN-POSITIVE MYELOPROLIFERATIVE NEOPLASMS IS COUNTERED BY HETEROCLITIC PEPTIDE CANCER VACCINATION}

'Mathieu Gigoux*, ${ }^{1}$ Roberta Zappasodi, ${ }^{1} J o s e p h$ Park, ${ }^{2}$ Cansu Cimen Bozkus, 'Levi Mangarin, 'David Redmond, 'Svena Verma, 'Sara Schad, 'Mariam George, ${ }^{1}$ Diyya Venkatesh, ${ }^{1}$ Arnab Ghosh, 'Zaki Molvi, ${ }^{3}$ Baransel Kamaz, ${ }^{3}$ Anna Marneth, ${ }^{3}$ William Duke, ${ }^{4}$ Matthew Leventhal, ${ }^{3}$ Max Jan, ${ }^{3}$ Vincent Ho, ${ }^{3}$ Gabriela Hobbs, ${ }^{5}$ Trine Knudsen, ${ }^{5}$ Vibe Skov, ${ }^{5}$ Lasse Kjễir, ${ }^{6}$ Thomas Larsen, ${ }^{6}$ Dennis Hansen, ${ }^{3}$ R. Lindsley, ${ }^{5}$ Hans Hasselbalch, ${ }^{7}$ Jacob Grauslund, ${ }^{7}$ Mads Andersen, ${ }^{7}$ Morten Holmstrom, ${ }^{1}$ Timothy Chan, ${ }^{1}$ Raajit Rampal, ${ }^{1}$ Omar Abdel-Wahab, ${ }^{2}$ Nina Bhardwaj, ${ }^{3}$ Ann Mullally, ${ }^{1}$ Jedd Wolchok, ${ }^{1}$ Taha Merghoub. ${ }^{1}$ Memorial Sloan Kettering Cancer Center, New York, NY, United States; ${ }^{2}$ Icahn School of Medicine at Mount Sinai, New York, United States; ${ }^{3}$ Harvard Medical School, Boston, United States; ${ }^{4}$ Broad Institute, Cambridge, MA, United States; ${ }^{5}$ Zealand University Hospital, Roskilde, Denmark; ${ }^{6}$ Odense University Hospital, Odense, Denmark; ${ }^{7}$ University of Copenhagen, Copenhagen, Denmark

Background The majority of JAK2V617F-negative myeloproliferative neoplasms (MPN) have disease-initiating frameshift mutations in calreticulin (CALR) resulting in a common novel C-terminal mutant fragment (CALRMUT), representing an attractive source of neoantigens for cancer vaccines. However, studies have shown that CALRMUT-specific $\mathrm{T}$ cells are rare in CALRMUT MPN patients, but the underlying reasons for this phenomenon are unknown. We speculate that this is due to an increased chance of immune-mediated tumor rejection by individuals expressing one of these MHC-I alleles such that the disease never clinically manifests. As a consequence of this MHC-I allele restriction, we reasoned that CALRMUT MPN patients would not efficiently respond to cancer vaccines composed of the CALRMUT neoantigen, but could do so when immunized with a properly epitope-optimized CALRMUT heteroclitic peptide vaccine approach.

Methods We examined MHC-I allele frequency in CALRMUT MPN patients from two independent cohorts to identify under-represented MHC-I alleles. These MHC-I alleles were assessed for their ability to bind to CALRMUT-derived peptides using NetMHC and were subsequently validated experimentally in healthy donors and in CALRMUT MPN patients having received a CALRMUT cancer vaccine (clinical trial NCT03566446) to determine if these MHC-I were potentiating immunogenicity against the CALRMUT antigen. Epitopeoptimized heteroclitic variants of the CALRMUT neoantigen were identified and tested experimentally in vitro in human PBMCs and in vivo in mice for their ability to mount an immune response against the non-modified CALRMUT neoantigen.

Results We observed that MHC-I alleles that present CALRMUT neoepitopes with high affinity are under-represented in CALRMUT MPN patients. Heteroclitic CALRMUT peptides specifically designed for CALRMUT MPN patient MHC-I alleles efficiently elicited a cross-reactive CD8 $+\mathrm{T}$ cell response in human PBMC samples otherwise unable to respond to the matched weakly immunogenic CALRMUT native peptides. We also modeled this effect in mice and observed that C57BL/6J mice, which are unable to mount an immune response to the human CALRMUT fragment, can mount a cross-reactive CD8 $+\mathrm{T}$ cell response against a CALRMUT-derived peptide upon heteroclitic peptide immunization and this was further amplified by combining the heteroclitic peptide vaccine with blockade of the immune checkpoint molecule PD-1.

Conclusions Our study shows that MHC-I alleles able to present CALRMUT neoepitopes are under-represented in CALRMUT MPN patients, demonstrating that MHC-I haplotype is a major mechanism of passive immune-evasion in
CALRMUT MPN. However, we show that a cancer vaccine composed of heteroclitic variants of the CALRMUT antigen could overcome this limitation.

Ethics Approval Approval was obtained for the use of patientderived specimens and access to clinical data extracted from patient charts by the Institutional Review Boards at Memorial Sloan Kettering Cancer Center, the Dana-Farber Cancer Institute and the Massachusetts General Hospital, as well as by the Danish Regional Science Ethics Committee.

http://dx.doi.org/10.1136/jitc-2021-SITC2021.772 